\title{
Three-dimensional seed reconstruction from an incomplete data set for prostate brachytherapy
}

\author{
Sreeram Narayanan ${ }^{1}$, Paul S Cho ${ }^{1,2}$ and Robert J Marks II $^{3}$ \\ ${ }^{1}$ Department of Electrical Engineering, University of Washington Seattle, WA 98195-2500, USA \\ 2 Department of Radiation Oncology, University of Washington School of Medicine Seattle, \\ WA 98195-6043, USA \\ ${ }^{3}$ Department of Engineering, Baylor University Waco, TX 76798-7356, USA \\ E-mail: psncho@u.washington.edu
}

Received 14 April 2004

Published 16 July 2004

Online at stacks.iop.org/PMB/49/3483

doi:10.1088/0031-9155/49/15/012

\begin{abstract}
Intra-operative dosimetry in prostate brachytherapy requires 3D coordinates of the implanted, radioactive seeds. Since CT is not readily available during the implant operation, projection $\mathrm{x}$-rays are commonly used for intra-operative seed localization. Three x-ray projections are usually used. The requirement of the current seed reconstruction algorithms is that the seeds must be identified on all three projections. However, in practice this is often difficult to accomplish due to the problem of heavily clustered and overlapping seeds. We have developed an algorithm that permits seed reconstruction from an incomplete data set. Instead of all three projections, the new algorithm requires only one of the three projections to be complete. Furthermore, even if all three projections are incomplete, it can reconstruct $100 \%$ of the implanted seeds depending on how the undetected seeds are distributed among the projections. The method utilizes the principles of epipolar imaging geometry and pseudo-matching of the undetected seeds. The algorithm was successfully applied to a large number of clinical cases where seeds imperceptibly overlap in some projections.
\end{abstract}

\section{Introduction}

Intra-operative dosimetry requires the computation of the 3D location of the implanted seeds. Since CT (Roy et al 1993, Brinkmann and Kline 1998) is not readily available during the implant operation, projection x-rays are commonly used for intra-operative seed localization (Tubic et al 2001, Gong et al 2002, Todor et al 2003). Three x-ray projections are usually needed. One significant limitation of the present reconstruction algorithms is their inability to reconstruct from an incomplete data set. That is, they require all the implanted seeds $(N)$ be 
identified in every projection. However, in practice, due to superposition of seeds, it is often impossible to detect all $N$ seeds on every projection. We propose a method to overcome this problem.

Calculation of 3D seed coordinates is a culmination of various steps starting with image capture, seed segmentation and reconstruction. When seed images are heavily clustered, the segmentation algorithm may fail to uniquely identify all the seeds on all three projections. Human operator can help find the seeds that were left undetected by the segmentation algorithm. However, in some cases even with operator intervention it is impossible to locate all the seeds. Thus, there is a need for a seed reconstruction algorithm that can handle incomplete data sets.

Siddon and Chin (1985) proposed a two-film reconstruction algorithm but had the restriction that if the two films have $N_{1}$ and $N_{2}$ seeds each with $N_{1}>N_{2}$, the number of reconstructed seeds will be $N_{2}$. Upon extending this algorithm to three films only the minimum number of seeds identifiable on a single film can be reconstructed under the condition that all three films are used for reconstruction. Biggs and Kelly (1983) proposed a reconstruction algorithm using the $y$-coordinate values as a pre-screening to reduce matching complexity. The $y$-axis is parallel to the axis of rotation of the gantry. Here again the problem of undetected seeds is left un-addressed and, hence, the algorithm cannot reconstruct all the implanted seeds successfully if some seeds are undetected. Altschuler and Kassaee (1997) proposed a permutation-sampling method for matching the seeds. The number of seeds reconstructed, however, is restricted by the number of unique triplets that can be identified, which essentially is the minimum number of seeds identified on a view. This can potentially lead to mismatches in the reconstruction if, for example, two views have one undetected seed each but do not correspond to the same 3D seed. Other projection x-ray-based seed reconstruction algorithms (Amols and Rosen 1981, Altschuler et al 1983, Jackson 1983, Rosenthal and Nath 1983, Tubic et al 2001, Narayanan et al 2002, Todor et al 2003) do not address the problem of undetected seeds. It is usually assumed that this problem does not occur or is rare. However, experience in our clinic shows that the problem of undetected seeds occurs in over $50 \%$ of the implant cases.

Lam et al (2004) developed a Hough transform based seed reconstruction algorithm that handles incomplete data sets. The algorithm assumes the use of more than three views and traces the path of a seed over multiple projections. Unlike the conventional approaches, where the 2D seed points are back-projected to reconstruct the 3D seed, the Hough transform assumes a point in 3D space and forward-projects it onto the detector plane to determine if the projected point coincides with a seed image. Those $3 \mathrm{D}$ points that accumulate high coincident counts are considered as the 3D locations of the seeds. However, the algorithm requires relatively large number of projections in the order of 10 and is not practical unless one has access to a fast image acquisition device.

Narayanan et al (2002) introduced a fast cross-projection algorithm for reconstruction of seeds (Fast-CARS) that performs reconstruction by reducing the matching complexity. The line connecting a seed image to the source is calculated and the projection of this line is obtained on another view. A search restriction band of certain width centred on the projected line is formed on this view. The candidate matching seed for the seed on the first view will lie in this band. The current version of Fast-CARS however cannot handle incomplete data sets.

We present a new algorithm that permits seed reconstruction from incomplete data sets. The algorithm requires that the implanted seeds $(N)$ be detected in only one of the three projections. Furthermore, even if none of the views has identified all $N$ seeds, reconstruction is possible depending on how the unidentified seeds are distributed among the projections. 


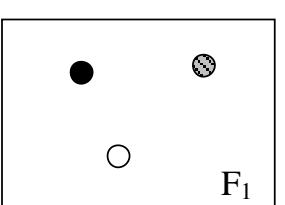

$\mathrm{F}_{1}$

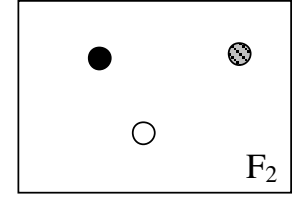

(a)
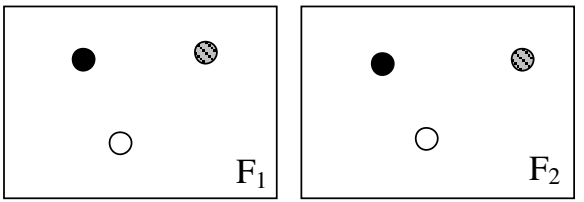

(b)
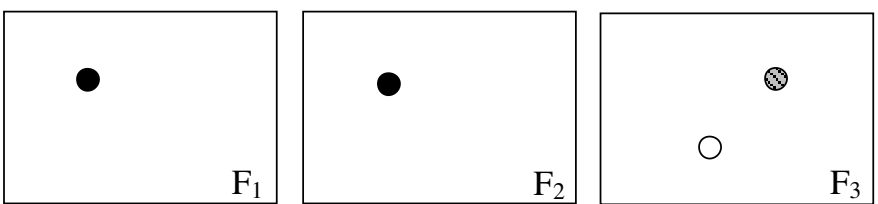

(c)

Figure 1. (a) An example to illustrate the pseudo-seed-matching concept. Consider three implanted seeds such that all three of them are visible on two views $\left(F_{1}\right.$ and $\left.F_{2}\right)$ and the third view $\left(F_{3}\right)$ has an undetected seed and thus only two are visible. (b) In order to perform seed matching and reconstruction, duplicate all the seeds in the view with undetected seeds, i.e. $F_{3}$. Before duplication, the numbers of seeds were $\{3,3,2\}$. After duplication they are $\{3,3,4\}$. (c) Match and reconstruct all seeds such that only as many duplicated seeds are chosen as are undetected in the view. In our example, only one duplicated seed will be chosen along with the two original seeds in $F_{3}$. The two seeds whose projections were visible on all three views are removed from consideration in (c) for ease of understanding. The solid circle in the first two views indicates the seed whose projection was undetected on the third view. One of the two doubles is a candidate for a match for this particular seed and is chosen based on minimum cost.

\section{Methods}

\subsection{Pseudo-seed-matching strategy}

The overall strategy for handling the incomplete data set is described using a simple example shown in figure 1. Consider a case where there are only three implanted seeds. All three seeds are visible on two of the three projections, $F_{1}$ and $F_{2}$. On the third view, $F_{3}$, only two out of the three seeds are visible. The third seed is obscured by one of the two visible seeds and is undetected (figure 1(a)). However, we know that the undetected seed coincides with one of the two visible seeds on projection $F_{3}$. Therefore, one of the two seeds is allowed to match twice. Since we do not know which one of the two coincides with the undetected seed, we duplicate both seeds (figure 1(b)). Subsequently, the reconstruction problem will be approached as a set of $\{3,3,4\}$ seeds with the knowledge that there are three seeds and thus only three unique triplets can be formed during the seed matching process of seed reconstruction. While performing the seed matching, care must be taken such that only as many doubles are chosen as are undetected in that view. In our example, there is one undetected seed in $F_{3}$ and thus only one of the two doubles will be chosen. Two seeds from $F_{1}$ and $F_{2}$ will match with the original two seeds in $F_{3}$ and the third seed will match with one of the duplicated, pseudo-seeds 
(figure 1(c)). The matching is performed such that the sum of the costs of the match set is minimal, where each match set is a combination of triplets of seeds in consideration. The cost for each triplet is the sum of the square of the distances from the reconstructed 3D point to the lines connecting each seed image to its source.

The problem of incomplete projection data can be categorized into three representative cases:

(a) $\{N, N, N-p\}$. Two out of three views have detected all $N$ seeds that were implanted. The third view has $p$ undetected seeds.

(b) $\{N, N-p, N-q\}$. One out of three views has identified all $N$ seeds. The other two have undetected seeds of arbitrary numbers, $p$ and $q$.

(c) $\{N-p, N-q, N-r\}$. None of the views has resolved all $N$ seeds. Every view is incomplete and has $p, q$ and $r$ undetected seeds.

Note that the orders of views in the above sets are arbitrary. For example, $\{N-p, N, N\}$, $\{N, N-p, N\}$ and $\{N, N, N-p\}$ all belong to the first data type (a) in the list. The pseudoseed-matching strategy was illustrated using the incomplete data type $\{N, N, N-p\}$. The same strategy applies to the data type $\{N, N, N-p\}$ as well. As will be explained later, the most demanding case $\{N-p, N-q, N-r\}$ can also be solved if it can be decomposed into $\{N, N, N-p\}$ and/or $\{N, N-p, N-q\}$ varieties.

We have applied the pseudo-matching technique to the previously reported fast crossprojection method (Narayanan et al 2002). The results indicate that because of the way the search restriction bands are formed, the Fast-CARS can handle only the incomplete data type $\{N, N, N-p\}$. Therefore, we have investigated an alternative method of forming the search restriction bands that will permit reconstruction from $\{N, N-p, N-q\}$ and $\{N-p, N-q, N-r\}$ data types.

\subsection{Epipolar geometry based reconstruction}

We first introduce the concept of epipolar geometry (Mundy and Zisserman 1992, Haralick and Shapiro 1993). Consider a plane containing two x-ray source positions and a 3D seed. This plane will intersect the detector planes and form lines. The $2 \mathrm{D}$ image of the seed on the detector planes will lie on these lines. The plane defined by the two $\mathrm{x}$-ray source positions and a 3D seed is called the epipolar plane. The line formed by the intersection of the epipolar plane and the detector plane is called the epipolar line. For each seed in 3D space we can form an epipolar plane. Thus constructed epipolar planes are hinged at the line connecting the two sources. Epipolar geometry is defined only over two projections. Therefore, constraint of co-planar imaging is required to extend this over three projections.

The property of epipolar geometry can be exploited to generate search restriction bands, which are used to accelerate the seed-matching process as described by Narayanan et al (2002). It is essentially a divide-and-conquer strategy, which performs seed matching for a sub-region of views at a time. Therefore, the strategy significantly reduces the computational dimensionality. The search restriction bands also help reduce the complexity of data incomplete conditions. For example, consider a case where the total number of implanted seeds, $N$, is 10 and the projection data set is $\{9,9,9\}$. This is the incomplete data type $\{N-p, N-q, N-r\}$, for which there is no solution for the undetected seed. The search restriction band may divide the seeds in each view into two groups, $\{5,5,4\}$ and $\{4,4,5\}$. Thus, the problem has been decomposed into $\left\{N_{1}, N_{1}, N_{1}-p\right\}$ and $\left\{N_{2}, N_{2}-p, N_{2}-q\right\}$, which are both solvable. In the above case, $N_{1}=N_{2}=5$ and $N_{1}+N_{2}=N$.

Consider a three-view reconstruction but two views at a time. Any plane pivoted about the line connecting two of the $\mathrm{x}$-ray sources will split the 3D seed volume into two sections 


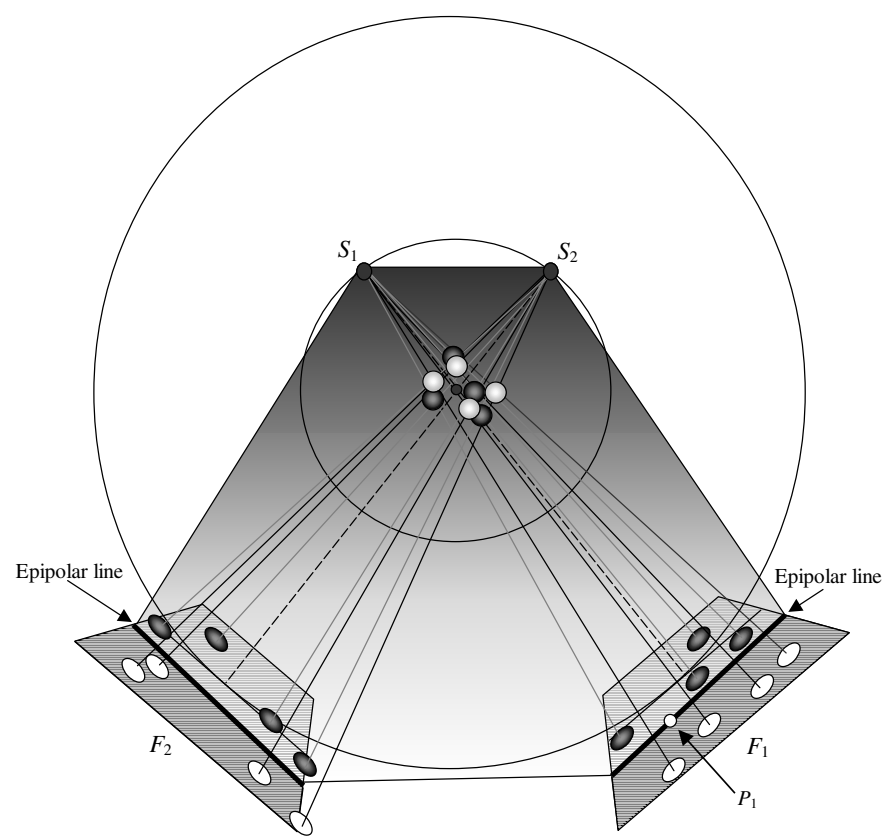

Figure 2. Definition of the epipolar plane and epipolar line. A plane pivoted around the line connecting the two sources will pass through the 3D volume of the seeds forming lines on the imaging planes. The lines split the $2 \mathrm{D}$ images of the seeds in the same proportion as the plane splits the 3D distribution of the seeds.

on either side of this plane (figure 2). As described above, the intersection of this plane with the two detector planes will form epipolar lines. In the absence of noise, epipolar lines will split the 2D images of the seeds on the detector plane in the exact same proportion as the epipolar plane splits the 3D seed volume. This proportion will be the same on either view. If the two views in consideration are $F_{1}$ and $F_{2}$ and if $P$ seeds are above the epipolar line on $F_{1}$ in a noiseless case and given that seeds are visible on both views, $P$ seeds will be above the epipolar line in $F_{2}$ as well. The epipolar plane will thus split the 3D volume into two sections of $P$ seeds and $N-P$ seeds, where $N$ is the total number of seeds implanted. This property of epipolar geometry is used to form different search restriction bands over three views, where if all seeds are detected, the bands contain equal number of seeds. The bands could be formed by progressively rotating the epipolar plane about the hinge defined by $S_{1}$ and $S_{2}$. For illustration, an epipolar plane is constructed using two sources $\left(S_{1}\right.$ and $\left.S_{2}\right)$ and a point, $p_{1}$, on the corresponding image planes. The second epipolar plane can be constructed using the same point $p_{1}$, its corresponding source $S_{1}$ and the source that was not previously considered, $S_{3}$.

In real world applications the data are expected to be noisy. This means that the epipolar plane will not necessarily split the seeds in two detector planes in equal portions at all times. The source of the noise includes patient motion. Thus motion estimation and correction must first be performed (Lam et al 2004). This leaves the random noise and uncertainty of the line, which can be solved by performing a low-pass filtering of data as described later.

The seed reconstruction algorithm for incomplete data set is outlined as follows:

1. Choose one view at random.

2. Compute the maximum and minimum $y$ coordinate values of the seeds on this view. The 
coordinate systems are the same as in Narayanan et al (2002). $Y$-axis on the detector plane is parallel to the axis of gantry rotation. $X$-axis is perpendicular to $Y$-axis.

3. Start with a $y$ value that corresponds to the minimum value obtained in step 2 and choose a point, $p_{1}$. This point could be $\left(x_{\text {val }}, y_{\text {min }}\right)$ where $x_{\text {val }}$ is any $x$-coordinate value. Point $p_{1}$ need not be a seed location.

4. Form two epipolar planes, one using $\mathrm{S}_{1}, \mathrm{~S}_{2}$ and $p_{1}$, and another with $\mathrm{S}_{1}, \mathrm{~S}_{3}$ and $p_{1}$.

5. Calculate the number of seeds below the epipolar line in each view.

6 . Increment the $y$ value by $\delta$ thus changing the value of $p_{1}$. Repeat steps 4 and 5. From step 3 , the point $p_{1}$ will change as $\left(x_{\mathrm{val}}, y_{\min }+\delta\right),\left(x_{\mathrm{val}}, y_{\min }+2 \delta\right)$ and in general has the value $\left(x_{\mathrm{val}}, y_{\min }+n \delta\right)$ till it is greater than $\left(x_{\mathrm{val}}, y_{\max }\right)$.

7. Repeat steps $4-6$ as long as the seed count stays the same for a pre-determined number of epipolar-line increments, $k$. The number of increments is a function of $\delta$, and increases as $\delta$ decreases. The size of $\delta$ is determined experimentally by changing the value of $\delta$ and noting the number of increments that gives a good division of seeds. If the seed count remains the same it signifies that an epipolar plane, which splits the seeds exactly, exists within this interval. In our case the interval is formed by points whose $y$-coordinate values vary from $y_{\min }+n \delta$ to $y_{\min }+(n+k) \delta$. This operation, in which the seed count is allowed to stabilize, corresponds to the low-pass filtering mentioned earlier. Figure 4(d) illustrates this step using a clinical case.

8. For the 2D seeds found within the search restriction band formed by the union of epipolar lines (steps 4-7), reconstruct the 3D seeds assisted by the pseudo-seed-matching technique:

(a) For an incomplete data set of the type $\{N, N, N-p\}$ duplicate the seeds in the view with $N-p$ seeds and reconstruct using the pseudo seed-matching technique.

(b) For the data type $\{N, N-p, N-q\}$ duplicate the seeds in incomplete views and reconstruct as many seeds as are present in the complete view, i.e., $N$ seeds. For example, consider three views with $\{10,9,8\}$ seeds. After duplicating the second and the third views we get $\{10,18,16\}$. Only ten unique triplets are allowed as dictated by the view containing all seeds. Among the nine duplicates in the second view only one will be chosen and nine original ones are used once. Among eight duplicates in the third view, two will be chosen and the eight original ones are used once.

(c) If the data type $\{N-p, N-q, N-r\}$ occurs within a given search restriction band, the undetected seeds cannot be recovered unless the number of seeds expected in the band is known.

9. Repeat steps 4-8, making sure that while taking the count of the seeds below the lines, the previously matched seeds are discarded. Each repetition of the steps corresponds to finding a new set of bands over three views, and reconstructing the seeds in this set.

\subsection{Problem of false positives}

In the process of automated seed segmentation, fragments of high-density objects such as bones and catheters may be falsely classified as seeds. There could also be cases where a single seed is classified as two closely overlapping seeds by the segmentation algorithm. These cases form what we call false positives and are treated separately. Normally, the false positives generated by the non-seed entities are easily identifiable and readily removed by the operator at the post-segmentation editing. However, the misclassification of a single seed as a double seed by the segmentation program is often difficult to rectify even by visual inspection. 
The false positives due to non-seed structures can be discriminated from the true positives by examining the cost associated with the seed reconstruction process. If a non-seed structure is falsely identified as a seed on one or two views, a duplicate coordinate of the seed nearest to the structure in the other view(s) will be used to form a triplet. In this case since the three $2 \mathrm{D}$ points that reconstruct the 3D point do not correspond to the same 3D object, the cost function value of this reconstruction will be very high. Therefore, a simple cost threshold can be used to eliminate such false positives. The method would fail if a 3D object of a non-seed origin generated the false positives on all three views. However, the probability of this happening is extremely rare. This is because the brightness of the high-density structures varies appreciably from projection to projection. Subsequently, the 2D seed detection algorithm tends to segment different parts of the high-density objects.

In the case where a single $2 \mathrm{D}$ seed is falsely determined as a double seed by the segmentation algorithm, the proposed method will find the corresponding seed in the other two projections. The seeds in the two projections will be duplicated erroneously and reconstruction algorithm will yield an extra seed. The cost threshold will not work in this case since three seed images were chosen to reconstruct the extra seed and hence will have a cost that is comparable to the rest of seed matching. However, we do have the knowledge that seeds need to be separated by a certain distance in 3D. If two seeds occupy the same 3D location, which will be the case in the above reconstruction scenario, we can safely assume that one of the seeds is wrong. This is especially the case if the algorithm reconstructs more seeds than have been implanted. Thus a 3D redundancy check can handle false positives occurring when a single seed is erroneously counted as two.

\section{Results and discussions}

The algorithm performance was evaluated on the clinical data. The fluoroscopy images were acquired with a radiotherapy simulator (SLS-14, Elekta Oncology Systems, Crawley, UK). The projection angles were separated by $15^{\circ}\left(165,180\right.$ and $\left.195^{\circ}\right)$. Two types of seeds, iodine-125 (6711, Amersham Health, Princeton, NJ, USA), and palladium-103 (TheraSeed, Theragenics Corporation, Buford, GA, USA), were imaged. Seed images were captured with the 6-inch image intensifier mode, corrected for spatial distortion, and automatically segmented (Cho 2000). The results were visually inspected by an operator and edited when necessary. Subsequently, the 2D seed data set was input to the proposed algorithm. In order to verify the accuracy of the reconstruction, the 3D results were forward-projected onto the original images and the deviations from the seed locations were calculated. If the errors were within $0.5 \mathrm{~mm}$, the reconstruction was considered to be successful.

Figure 3 shows an example of reconstruction from an incomplete data set of the type $\{N, N, N-p\}$. There were 84 seeds in total. Two views had all 84 seeds detected and the third view identified only 82 seeds. The reconstruction algorithm successfully reconstructed all 84 seeds. The reconstructed seed centroids were forward-projected on the view that had only 82 seeds identified. The arrows point to the overlapped seeds that were not detected by the segmentation algorithm, but reconstructed accurately.

Figures 4(a), (b) and (c) illustrate how the incomplete data set of the type $\{N-p, N-q$, $N-r\}$ can be simplified. In this example, a total of 37 seeds were implanted, of which $\{35,36,36\}$ were detected. However, observation of the three views reveals that the two undetected seeds on the first view (figure 4(a)) are located in the same search restriction band and the other two views provide complete data for the band. Therefore, for this particular band the data incompleteness is essentially of the type $\{N-p, N, N\}$. It is also noted that 


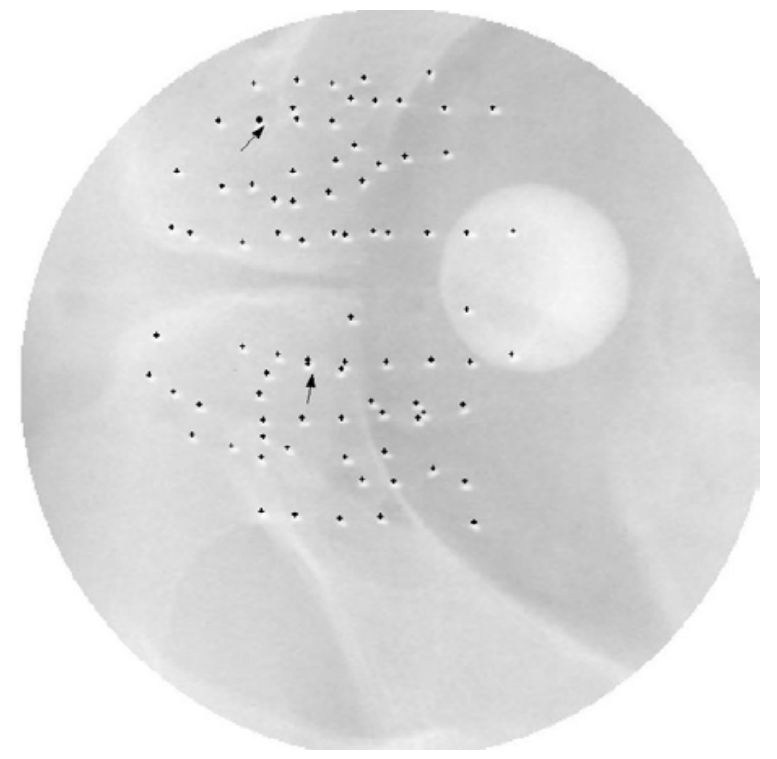

Figure 3. Seed reconstruction from an incomplete data set of the type $\{N, N, N-p\}$ or $\{84,84,82\}$. The algorithm reconstructed all 84 seeds. The results (3D seed centroids) were forward-projected onto the view that had identified only 82 seeds. The two overlapped seeds that were not detected by the seed segmentation algorithm, but reconstructed accurately, are indicated by the arrows.

the second (figure 4(b)) and the third view (figure 4(c)) together form a $\{N, N-p, N-q\}$ case for the lowest band, which is solvable.

Figure 4(d) shows the epipolar line counts between image pairs. Images in figures 4(a) and (b) form epipolar line pairs for the left column of the figure (views 1 and 2) and figures 4(b) and (c) form the pairs for the right column corresponding to views 2 and 3. The top plots show the set of all possible epipolar line counts, for each $\delta$ increment in the $y$ value. The bottom plots show the 'filtered' version of the epipolar line counts where only repeated counts are shown. The epipolar lines are formed bottom up, so in figures 4(a), (b) and (c), the first band corresponds to the bottom row of the seeds. Repeated counts indicated by the arrows are not visible in the top plots because they are right on top of each other. The first band, formed between views 1 and 2, has one undetected seed in view 2. This is indicated by the fact that the seed count is less than the equal count line and thus there is an undetected seed in the view corresponding to the $y$-axis of the plot. When comparing views 2 and 3, we see that they have the same count across all bands. This implies that there is an undetected seed in the first band of view 3 also. In the second band between views 1 and 2 a jump in seed count for view 2 is noticed. Now the value is over the equal count line, which means that there are two undetected seeds in view 1. Views 2 and 3 have the same count so there are no more undetected seeds in either of them.

Figures 5(a), (b) and (c) show another $\{N-p, N-q, N-r\}$ case. There were 109 seeds in total, only 108 of which were detected on each view. Conventional reconstruction algorithms would reconstruct only 108 seeds of which at least 3 would be mismatches. The seeds are unidentified in different places thus making this reconstruction process a multiple $\{N, N, N-p\}$ case. The proposed reconstruction algorithm detected all 109 seeds.

The algorithm was tested on 122 clinical cases (78 iodine and 44 palladium cases). As can be seen in table 1 of the 122 cases, 73 had view(s) with undetected seeds. The algorithm 


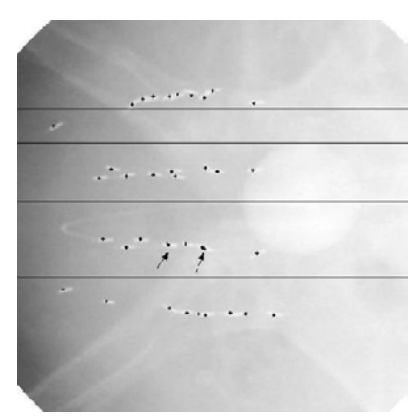

(a)

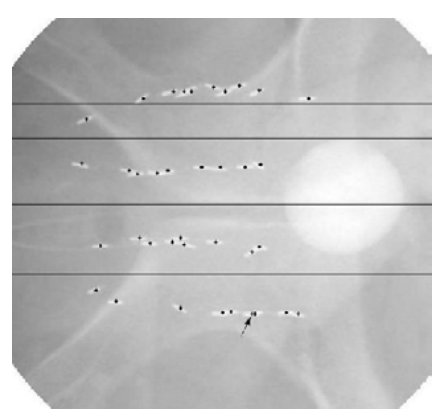

(b)

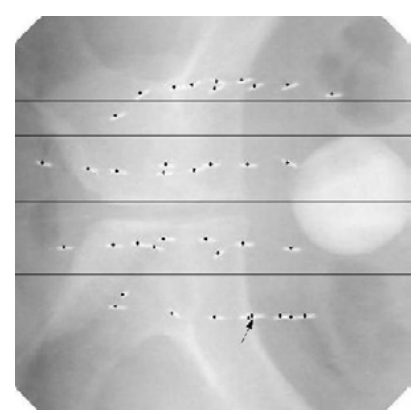

(c)

Set of all seed counts between Views $1 \& 2$

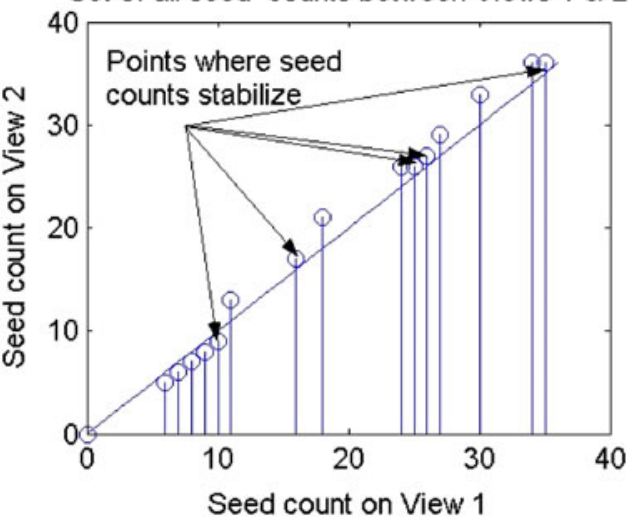

Set of all seed counts between Views $2 \& 3$

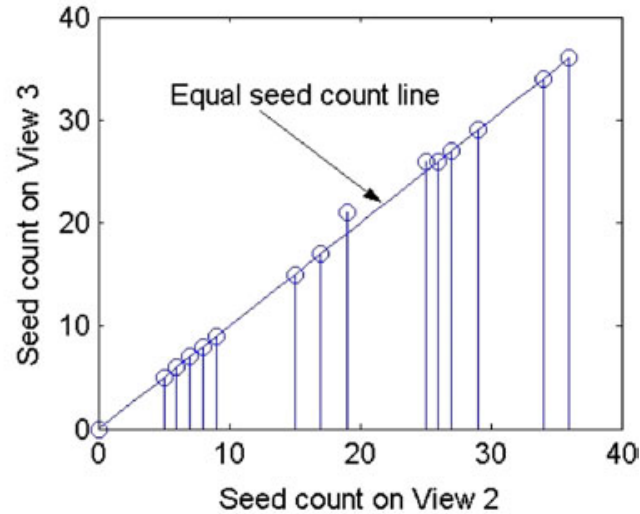

Seed counts after step 7 for Views 1 \& 2

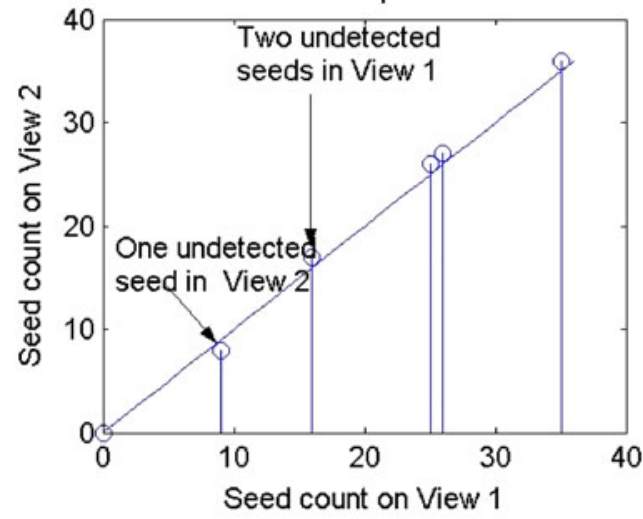

Seed counts after step 7 for Views $2 \& 3$

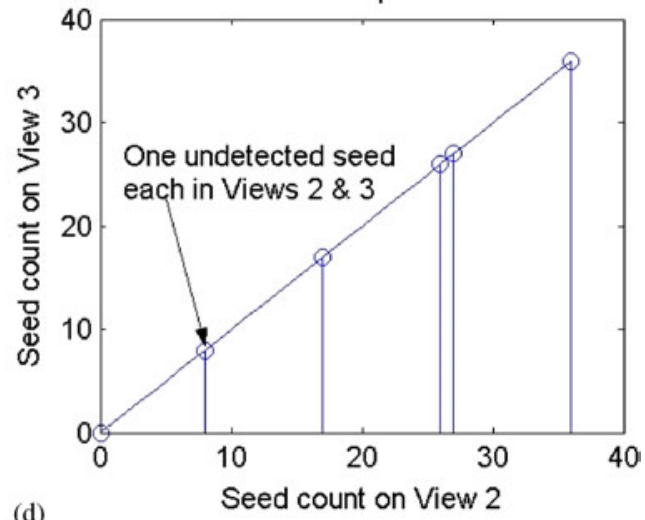

(d)

Figure 4. (a) 37 seeds were implanted in total and only 35 were detected on this view. Each arrow indicates two superposed seeds. Only one of the superposed seeds was detected in each cluster. However, since the two undetected seeds are visible in the other two views ((b) and (c)), for this particular search restriction band, the incomplete data type is $\{N, N, N-p\}$ which is solvable with the pseudo-seed-matching technique. (b), (c) The two views with an undetected seed in the same band. The arrow points to the undetected seed due to superposition. For this particular band, the incomplete data type is $\{N, N-p, N-q\}$, which is solvable. (d) Set of all seed counts, where view 1 corresponds to (a), view 2 corresponds to (b) and view 3 corresponds to (c). The top row corresponds to all sets of seed counts between views. The stabilized seed counts after step 7 are shown in the bottom rows. The undetected seeds can be noted by verifying if counts are above or below the equal seed count line. If two views have exactly the same seed counts, then they will lie on the line as shown in the bottom right figure. 


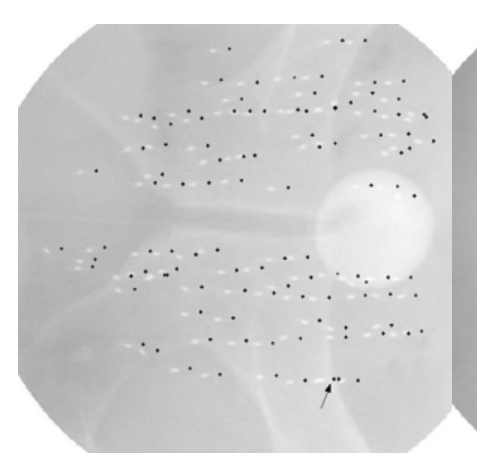

(a)

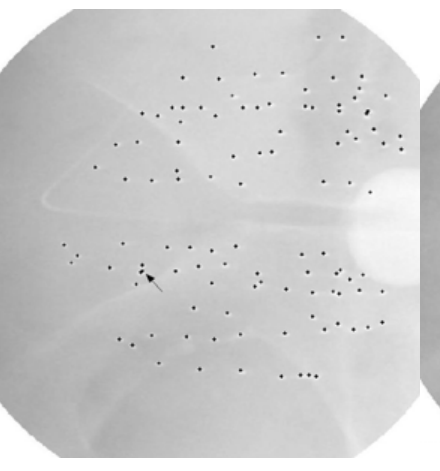

(b)

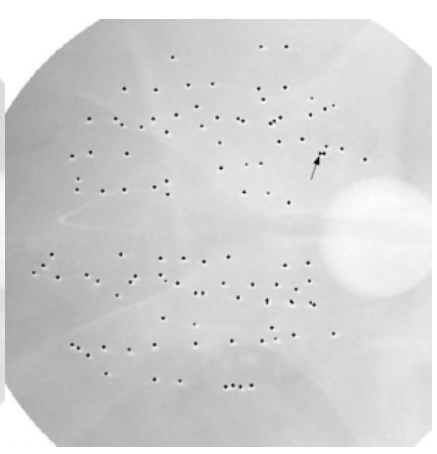

(c)

Figure 5. 109 seeds were implanted for this case. Fluoroscopy projections were acquired at 165,180 and $195^{\circ}$ from left to right. Only 108 seeds were detected on each view, thus it was a $\{N-p, N-q, N-r\}$ case. There was also a slight patient movement in this case which had to be corrected for before the reconstruction was performed. The patient moved between views (a) and (b). Views (b) and (c) were considered to be the reference. Hence the forward-projections of the reconstructed seed coordinates in view (a) are uniformly offset. The arrows indicate the undetected seeds, which were recovered and reconstructed by the algorithm.

Table 1. Results of the seed reconstruction classified according to the data type.

\begin{tabular}{|c|c|c|c|c|}
\hline \multirow[b]{2}{*}{ Data type } & \multicolumn{2}{|c|}{$\mathrm{I}-125$} & \multicolumn{2}{|c|}{ Pd-103 } \\
\hline & $\begin{array}{l}\text { Number of } \\
\text { implant } \\
\text { cases }\end{array}$ & $\begin{array}{l}\text { Number of cases } \\
\text { successfully } \\
\text { reconstructed }\end{array}$ & $\begin{array}{l}\text { Number of } \\
\text { implant } \\
\text { cases }\end{array}$ & $\begin{array}{l}\text { Number of cases } \\
\text { successfully } \\
\text { reconstructed }\end{array}$ \\
\hline$\{N, N, N\}$ & 37 & 37 & 12 & 12 \\
\hline$\{N, N, N-p\}$ & 23 & 23 & 12 & 12 \\
\hline$\{N, N-p, N-q\}$ & 14 & 14 & 10 & 10 \\
\hline$\{N-p, N-q, N-r\}$ & 4 & 4 & 10 & 6 \\
\hline Total & 78 & 78 & 44 & 40 \\
\hline
\end{tabular}

successfully reconstructed all the 78 iodine cases. Among the palladium cases, the algorithm reconstructed all cases of incomplete-data types $\{N, N, N-p\}$ and $\{N, N-p, N-q\}$. Out of the ten $\{N-p, N-q, N-r\}$ cases, the algorithm was able to reconstruct $100 \%$ of the implanted seeds for six cases. As for the four cases that were not successful, the algorithm correctly reconstructed 73/74, 100/102, 66/70 and 104/106 seeds without introducing any false positives, where $a / b$ implies that $b$ seeds were implanted and $a$ seeds were reconstructed. The percentages of undetected seeds for these cases were 1.4, 2.0, 5.7 and 1.9\%. It has been reported that the changes in implant quality, as measured in D90, V90 and tumour control probability, are negligible when the fraction of unlocalized seeds is in the order of $5 \%$ of the total implanted seeds (Lindsay et al 2003).

From table 1 it is evident that incomplete data sets occur with high frequency. In most cases the expected number of seeds in a band is known from one of the three views and hence the reconstruction can be performed using the pseudo-matching tactic. Only 4 of the 122 cases were of the $\{N-p, N-q, N-r\}$ type that could not be decomposed into solvable combinations of $\{N, N, N-p\}$ and $\{N, N-p, N-q\}$. Incomplete data problem occurs more often with palladium seeds as the small and rounded radio-opaque markers are difficult 
to segment when seeds overlap. In comparison, the long iodine seeds with high aspect ratios can be more easily resolved when clustered together.

Even though we have addressed a few cases where false positives could be eliminated, the combination of false positives and undetected seeds complicate the problem further. It might so happen that the false positive seed and the undetected seed are in the same band. The algorithm cannot identify the difference and will substitute the false positive seed for the seed that was not detected and perform reconstruction. A $\{N, N-p, N-q\}$ case with a false positive in the third view is actually a $\{N, N-p, N-q-1\}$ case in the absence of false positives. This problem needs to be solved by a different approach. It is thus best for the user to remove the obvious false positives in the seed-editing step and not guess to make up for the correct number of seeds.

The algorithm cannot reconstruct undetected seeds if they exist in the same search restriction band on all three views. The problem can be solved if we have prior knowledge of the number of seeds implanted within the band. This information is obtained from the number of seeds per needle track. Another way to get around the problem is to use more than three views and form epipolar line counts. Once this is done the three best views for each band can be chosen to perform reconstruction. In such a case we have a higher likelihood of having three complete image sets.

The current algorithm based on epipolar geometry requires co-planar imaging to perform reconstruction. Application of the pseudo-seed-matching method to fast cross-projection algorithm (Fast-CARS) (Narayanan et al 2002) can solve the problem of undetected seeds for non-coplanar imaging. However, the application is limited to the $\{N, N, N-p\}$ case. This is because the fast cross-projection algorithm forms search restriction bands in such a way that unequal numbers of seeds are enclosed in different views for a given band. If the search restriction bands in Fast-CARS can be formed in such a way that they have equal number of seeds per band in the case of no undetected seed, Fast-CARS will also be able to solve $\{N, N-p, N-q\}$ and some $\{N-p, N-q, N-r\}$ data types.

\section{Conclusion}

A new algorithm was presented that permits seed reconstruction from an incomplete data set. The algorithm requires only one of the three projections to be complete. Furthermore, even if all three projections are incomplete, it can reconstruct $100 \%$ of the implanted seeds depending on how the undetected seeds are distributed among the search restriction bands.

\section{Acknowledgments}

This work was supported in part by grants from the National Institutes of Health R21-CA89061 and the Department of Defense DAMD17-03-1-0033.

\section{References}

Altschuler M D, Findlay P A and Epperson R D 1983 Rapid, accurate, three-dimensional location of multiple seeds in implant radiotherapy treatment planning Phys. Med. Biol. 28 1305-18

Altschuler M D and Kassaee A 1997 Automated matching of corresponding seed images for three simulator radiographs to allow 3D triangulation of implanted seeds Phys. Med. Biol. 42 293-302

Amols H I and Rosen I I 1981 A three-film technique for reconstruction radioactive seed implants Med. Phys. 8 210-4 Biggs P J and Kelly D M 1983 Geometric reconstruction of seed implants using a three-film technique Med. Phys. $10701-5$ 
Brinkmann D H and Kline R W 1998 Automated seed localization from CT datasets of the prostate Med. Phys. 25 1667-72

Cho P S 2000 Computerized segmentation of clustered seeds in prostate brachytherapy Proc. 13th Int. Conf. on Computers in Radiotherapy ed W Schlegel and T Bortfeld (Heidelberg: Springer) pp 105-7

Cho P S, Narayanan S, Lam S T, Pathak S D, Tutar I B, Gong L, Kim Y and Wallner K E 2004 Dynamic brachytherapy: intra-operative dose optimization using dual-modality imaging Proc. 14th Int. Conf. Computers in Radiation Therapy ed B Yi, S Ahn, E Choi and S Ha (Seoul: Jeong Publishing) pp 10-3

Gong L, Cho P S, Han B H, Wallner K E, Sutlief S G, Pathak S D, Haynor D R and Kim Y 2002 Ultrasonography and Fluoroscopic Fusion for Prostate Brachytherapy Dosimetry Int. J. Radiat. Oncol. Biol. Phys. 54 1322-30

Haralick R M and Shapiro L G 1993 Computer and Robot Vision vol 2 (Reading, MA: Addison-Wesley)

Jackson D D 1983 An automatic method for localizing radioactive seeds in implant dosimetry Med. Phys. 10 370-2

Lam S T, Cho P S, Marks R J II and Narayanan S 2004 3D Seed reconstruction for prostate brachytherapy using hough trajectories Phys. Med. Biol 49 557-69

Lindsay P E, Van Dyk J and Battista J J 2003 Imaging uncertainties in prostate brachytherapy Med.Phys. 30 1897-908

Mundy J L and Zisserman A 1992 Geometric Invariance in Computer Vision (Cambridge, MA: MIT Press)

Narayanan S, Cho P S and Marks R J II 2002 Fast cross-projection algorithm for reconstruction of seeds in prostate brachytherapy Med. Phys. 29 1572-9

Rosenthal M S and Nath R 1983 An automatic seed identification technique for interstitial implants using three isocentric radiographs Med. Phys. 10 475-9

Roy J N, Wallner K E, Harrington P J, Ling C C and Anderson L L 1993 A CT-based evaluation method for permanent implants: application to prostate Int. J. Radiat. Oncol., Biol., Phys. 26 163-9

Siddon R L and Chin L M 1985 Two-film brachytherapy reconstruction algorithm Med. Phys. 12 77-83

Todor D A, Zaider M, Cohen G N, Worman M F and Zelefsky M J 2003 Intra-operative dynamic dosimetry for prostate implants Phys. Med. Biol. 48 1153-71

Tubic D, Zaccarin A, Beaulieu L and Pouliot J 2001 Automated seed detection and three-dimensional reconstruction. II. Reconstruction of permanent prostate implants using simulated annealing Med. Phys. 28 2272-9 